\title{
Nontrivial behavior of the Fermi arc in the staggered-flux ordered phase
}

\author{
Koichi Hamada and Daijiro Yoshioka \\ Department of Basic Science, University of Tokyo, 3-8-1 Komaba, Tokyo 153-8902, Japan
}

(October 16, 2003)

\begin{abstract}
The doping and temperature dependences of the Fermi arc in the staggered-flux, or the $d$-density wave, ordered phase of the $t-J$ model are analyzed by the $\mathrm{U}(1)$ slave boson theory. Nontrivial behavior is revealed by the self-consistent calculation. At low doped and finite-temperature region, both the length of the Fermi arc and the width of the Fermi pocket are proportional to $\delta$ and the area of the Fermi pocket is proportional to $\delta^{2}$. This behavior is completely different from that at the zero temperature, where the area of the Fermi pocket becomes $\pi^{2} \delta$. This behavior should be observed by detailed experiments of angle-resolved photoemission spectroscopy in the pseudogap phase of high- $T_{\mathrm{c}}$ cuprates if the pseudogap phase is the staggered-flux ordered phase.
\end{abstract}

74.72.-h, 71.27.+a, 74.20.Mn

The essence of the pseudogap phase of the high- $T_{c}$ superconductors in the low-doping region has not been clarified. There are several theoretical proposals for the origin of this phase, [1-11] and among them staggered flux state $[12,13]$ characterized by staggered orbital current is one of the most promising candidate. This state successfully explains various aspects of the pseudogap phase, such as weak magnetic signals caught by recent neutron scattering experiments of underdoped $\mathrm{YBa}_{2} \mathrm{Cu}_{3} \mathrm{O}_{6}$ (YBCO) [14,15], alternating magnetic signals in the vortex core observed by recent muon spin rotation experiments of underdoped YBCO [16], structure of underdoped vortex, [17] lack of specific-heat anomaly, [18,19] competition with $d$-wave pairing, [20-22] gap evolution, [22] etc. In particular, it has been shown that the Fermi arc observed by the angle-resolved photoemission spectroscopy (ARPES) experiments [23] in the pseudogap phase can be explained by the staggered flux state. $[22,24]$ Here what is meant by the Fermi arc is a gapless region near $(\pi / 2, \pi / 2)$, which does not form a closed loop.

It is desirable, however, to have further support for the staggered-flux state. In a previous paper [22] we have noticed that the theoretically obtained Fermi arc, or Fermi surface, at finite temperature is much smaller than that of a naive expectation that it is given by the density of doped holes. This means that the Fermi arc has strong temperature dependence, and hence it should have nontrivial doping dependence at finite temperatures. Thus if such nontrivial behavior of the Fermi arc is observed experimentally, it will give additional support for the staggered flux state. In order to make the comparison between the theory and experiment meaningful, we have investigated the $t-J$ model, and obtained detailed temperature and doping dependence of the Fermi surface in the staggered flux phase.

We have investigated the $t-J$ model based on the $\mathrm{U}(1)$ slave boson theory. In the present paper we set the parameter $t / J=3$ that is relevant for high- $T_{\mathrm{c}}$ superconductors and the unit lattice length $a=1$. The exchange energy $J$ is conventionally considered to be around $1500 \mathrm{~K}$.

We firstly review the proposed phases and the Fermi surfaces in the U(1) slave boson $t-J$ model. The uniform RVB (resonating valence bond) phase, where hopping order parameters are real and uniform, is thought to represent the anomalous metal (non Fermi-liquid) phase above the Bose-condensation (BC) temperature of holons [25] and the Fermi-liquid phase below the $\mathrm{BC}$ temperature. In this phase, a large Fermi surface is formed whose area is proportional to $1-\delta$ and Luttinger theorem is satisfied, where $\delta$ is hole concentration. The $d$-wave RVB phase, where spinons form $d_{x^{2}-y^{2}}$-wave pairs, and $d$-wave superconducting ( $d$-wave RVB phase with BC of holons) phase $[26,27]$ have always pointlike Fermi surface which exists in the nodal direction. In the staggered-flux ordered phase, the Fermi surface forms an arc [22] consistent with the ARPES experiments [23] in the pseudogap phase. To be more precise, the Fermi arc is a natural feature only in the $\mathrm{U}(1)$ slave boson theory. On the other hand, in the $\mathrm{SU}(2)$ slave boson theory, artificial introduction of the phenomenological interactions are needed for reproducing a Fermi arc because the chemical potential is always zero due to the $\mathrm{SU}(2)$ symmetry even at finite doping. [1]

In ordinary metals, the Fermi surface is almost temperature-independent because even room temperature is much lower than its Fermi energy. On the contrary, we reveal that there is strong and nontrivial doping and temperature dependences of the Fermi arc in the staggered flux ordered phase in this paper. This is because the low-energy excitation in this phase is described by a $(2+1)$-dimensional anisotropic masslessDirac-Fermion around the $(\pi / 2, \pi / 2)$ (and its symmetric points) with finite chemical potential. The expanded spectrum around the $(\pi / 2, \pi / 2)$ is given as the following:

$$
E_{\mathbf{k} \pm} \approx \pm \sqrt{2} \sqrt{X^{2} \tilde{k}_{+}^{2}+Y^{2} \tilde{k}_{-}^{2}}-\mu
$$

where $X=2 t x_{\mathrm{h}}+(3 J / 4) x_{\mathrm{s}}, \quad Y=2 t y_{\mathrm{h}}+(3 J / 4) y_{\mathrm{s}}$, $\tilde{k}_{ \pm}=\left(\tilde{k}_{x} \pm \tilde{k}_{y}\right) / \sqrt{2}, \tilde{k}_{i}=k_{i}-\pi / 2(i=x, y)$, and $x_{\mathrm{s}}$, $y_{\mathrm{s}}, x_{\mathrm{h}}$, and $y_{\mathrm{s}}$ are order parameters whose details will be elaborated later. Since $\mu<0, E_{\mathbf{k}-}=0$ gives the Fermi arc. As $X>Y$ in the staggered-flux phase, the 
zero-energy line of the fermion forms an ellipse at finite doping (Fig. 1). The Fermi surface can be considered as an arc although the zero-energy line of the fermion forms an ellipse. It is because the intensity of the spectral function is small in the outer region (where $\left|k_{x}\right|+\left|k_{y}\right| \geq \pi$ ). [22]

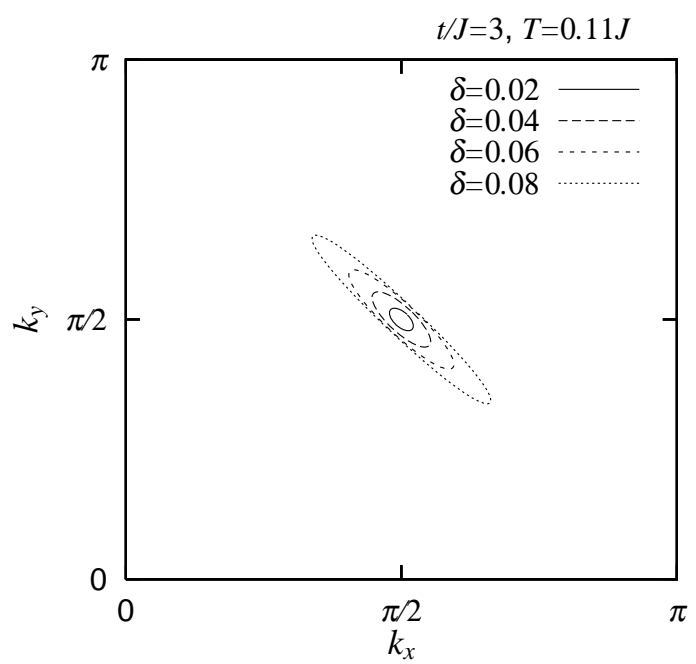

FIG. 1. The doping dependence of the zero-energy line of the fermion at $T=0.11 \mathrm{~J}$. The first quadrant of the Brillouin zone is shown. The Fermi surface can be considered as an arc although the zero-energy line of the fermion forms an ellipse. (Ref. 22)

First, we show doping-dependence of three characteristic quantities of the Fermi surface shown in Fig. 1: (i) the major axis $l_{1}$ in the $(0, \pi)-(\pi, 0)$-direction, (ii) the minor axis $l_{2}$ in the $(0,0)-(\pi, \pi)$-direction, and (iii) the area of the Fermi pocket $S$.

At zero temperature (Fig.2 (a)) the area of the Fermi pocket $S$ is $\pi^{2} \delta$, which is determined by the selfconsistent equation for total fermion number. The $l_{1}$ and $l_{2}$ are proportional to $\sqrt{\delta}$ near half-filling because the amplitude of the flux is near $\pi$, i.e., $X \approx Y$, but in other region the dependence is not $\sqrt{\delta}$. When the amplitude of the staggered flux decreases, the rate $Y / X$ decreases and the ellipse of the energy-contour develops to $\tilde{k}_{x}=-\tilde{k}_{y}$ $((0, \pi)-(\pi, 0))$ direction. When the staggered-flux order is completely destroyed, a large Fermi-surface whose area is proportional to $1-\delta$ is formed and $l_{1}$ becomes roughly $\sqrt{2} \pi$.

At finite temperature, the behavior is different. In Fig. 2 (b), we show a characteristic doping dependence at finite temperature, $T=0.11 \mathrm{~J}$, which corresponds to about $165 \mathrm{~K}$. Both the major axis $l_{1}$ and the minor axis $l_{2}$ develop linearly to $\delta$ and the area $S$ is proportional to $\delta^{2}$ at lightly doped region. As doping increases, $l_{2}$ saturates rapidly and only $l_{1}$ increases, i.e., the Fermi arc develops only in the $(0, \pi)-(\pi, 0)$-direction.
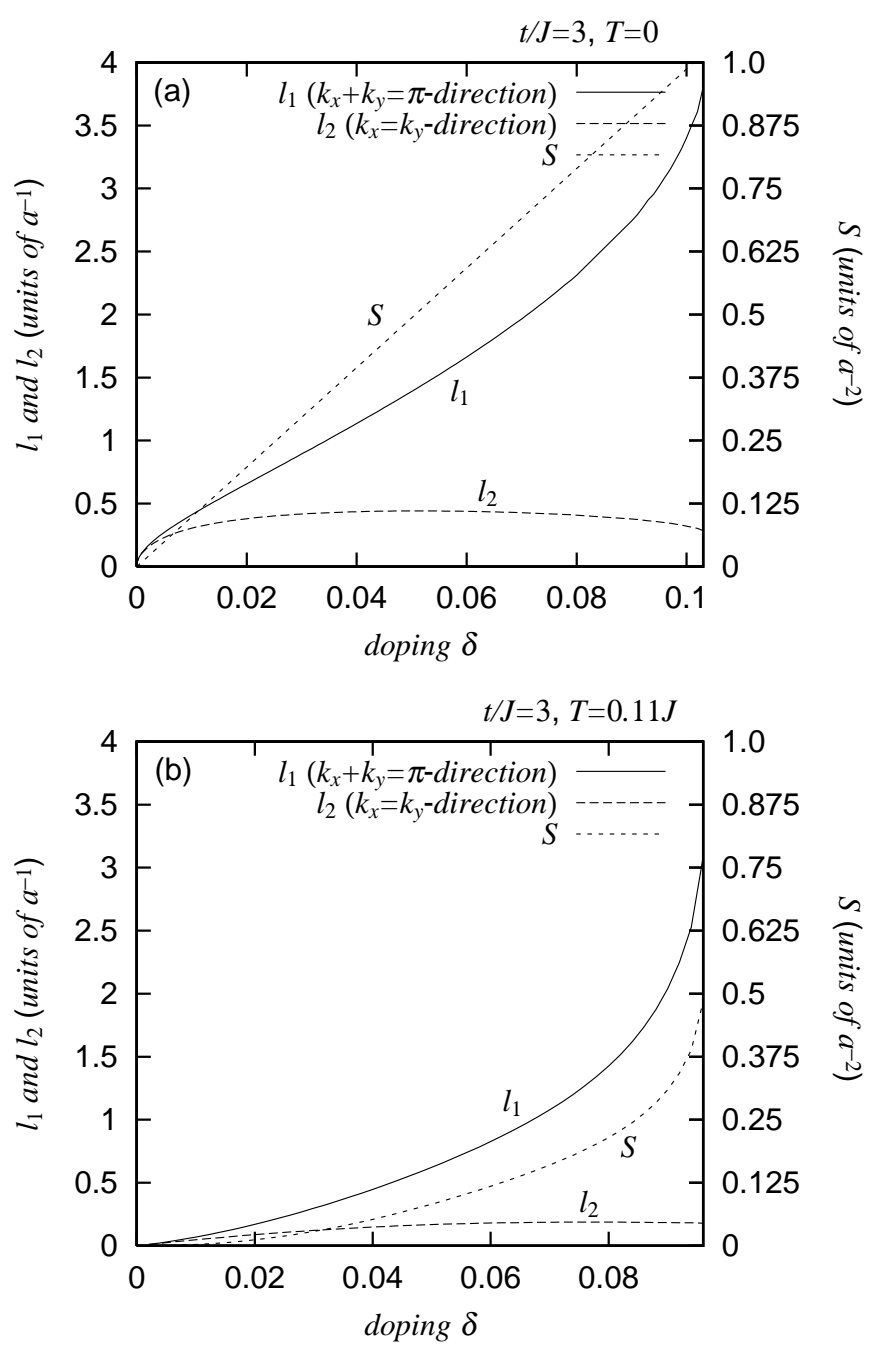

FIG. 2. A characteristic doping-dependence of the Fermi arc at low and high temperature region: The dependences of the major axis $l_{1}$, the minor axis $l_{2}$, and the area $S$ at $T=0$ (a) and $0.11 J$ (b) are shown.

This theoretically obtained Fermi arc, or Fermi surface, at finite temperature (Fig. 2(b)) is much smaller than that of a naive expectation that it is given by the density of doped holes. This means that the Fermi arc has strong temperature dependence. The temperature dependence at $\delta=0.01$ is shown in Fig. 3. When temperature increases, the area $S$ becomes smaller.

In the staggered flux phase, the low-energy-excitation is described by an anisotropic massless-Dirac-Fermion with finite chemical potential and the shape of the spectrum is conical. As the density of states of the lower cone, where the Fermi level lies, decreases rapidly at higher energy, the chemical potential increases as the temperature becomes higher, and the cross section of the cone becomes smaller. This is the origin of the strong temperature dependence of the Fermi arc at low temperature. The length of the Fermi arc stops to decrease and be- 
gins to increase when temperature increases much higher, as the staggered flux order begins to be destroyed with approaching the transition temperature to the uniform RVB phase. Finally, at the transition temperature, a large Fermi-surface is formed.

When the doping increases, this temperature dependence of the Fermi arc wholly becomes weaker. It is because the amplitude of the staggered flux order decreases and the conical spectrum becomes flatter. As the minimum value of $l_{1}$ becomes larger and $l_{1}$ is always fixed roughly $\sqrt{2} \pi$ at transition temperature, the sharpness of the increase near the transition point also becomes softer. These strong temperature dependences also make the doping dependence of the Fermi arc nontrivial.

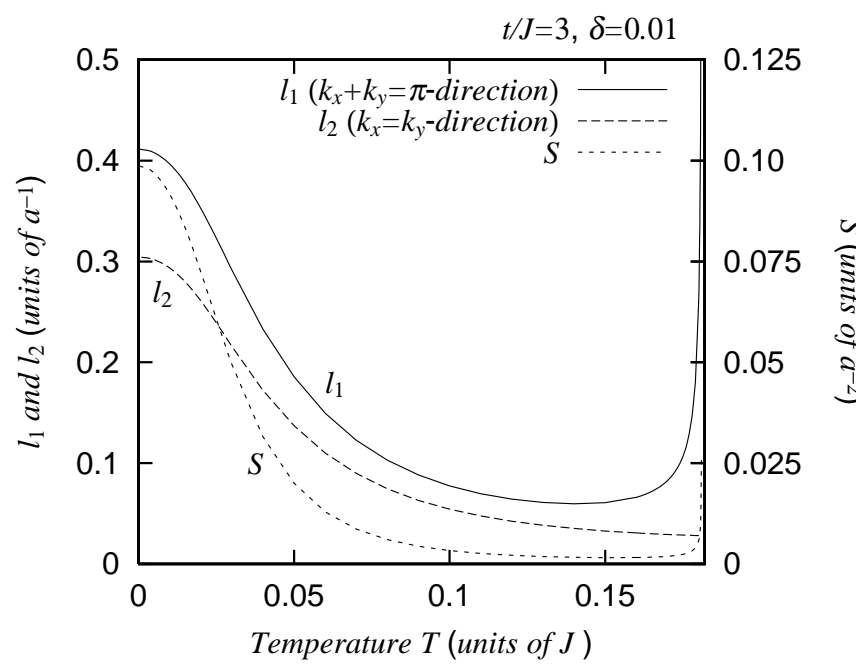

FIG. 3. A characteristic temperature-dependence of the Fermi arc: The dependence of the major axis $l_{1}$, the minor axis $l_{2}$, and the area $S$ at $\delta=0.01$ are shown.

In the following we report the details of our calculation. We follow the same path taken in Ref. 22 while considering only the staggered-flux state here, not considering the $d$-wave RVB or the $d$-wave superconducting state.

We analyze the two-dimensional $t-J$ model on a square lattice: $H=-t \sum_{\langle i, j\rangle} \sum_{\sigma} \boldsymbol{P}\left(c_{i \sigma}^{\dagger} c_{j \sigma}+\right.$ H.c. $) \boldsymbol{P}+$ $J \sum_{\langle i, j\rangle} \boldsymbol{S}_{i} \cdot \boldsymbol{S}_{j}$ that describes doped Mott insulators which is essential of high- $T_{c}$ superconductors. Here, $\langle i, j\rangle$ represents sum over the nearest-neighbor sites and $\boldsymbol{P}$ is a projection operator to no doubly occupied state, $\boldsymbol{S}_{i}$ represents spin- $1 / 2$ operator $\boldsymbol{S}_{i}=\frac{1}{2} c_{i \sigma}^{\dagger}(\boldsymbol{\sigma})_{\sigma \sigma^{\prime}} c_{i \sigma^{\prime}}$, where $\boldsymbol{\sigma}=\left(\sigma_{1}, \sigma_{2}, \sigma_{3}\right)$ are Pauli matrices. In the $\mathrm{U}(1)$ slave boson theory, the physical electron operator $c_{i \sigma}$ is described by a product of an auxiliary spin-1/2 neutral fermion operator $f_{i \sigma}$ called spinon and an auxiliary spinless charged boson operator $b_{i}$ called holon; $c_{i \sigma}=b_{i}^{\dagger} f_{i \sigma}$ with a constraint $b_{i}^{\dagger} b_{i}+\sum_{\sigma} f_{i \sigma}^{\dagger} f_{i \sigma}=1$.

For the mean-field solution, we considered both the staggered flux order of spinons and that of holons: $\left\langle f_{i+\hat{x} \sigma}^{\dagger} f_{i \sigma}\right\rangle=\chi \mathrm{e}^{i(-1)^{i} \phi_{\mathrm{s}} / 4}=x_{\mathrm{s}}+i(-1)^{i} y_{\mathrm{s}},\left\langle f_{i+\hat{y} \sigma}^{\dagger} f_{i \sigma}\right\rangle=$ $\chi \mathrm{e}^{-i(-1)^{i} \phi_{\mathrm{s}} / 4}=x_{\mathrm{s}}-i(-1)^{i} y_{\mathrm{s}},\left\langle b_{i+\hat{x}}^{\dagger} b_{i}\right\rangle=B \mathrm{e}^{i(-1)^{i} \phi_{\mathrm{h}} / 4}=$ $x_{\mathrm{h}}+i(-1)^{i} y_{\mathrm{h}}$, and $\left\langle b_{i+\hat{y}}^{\dagger} b_{i}\right\rangle=B \mathrm{e}^{-i(-1)^{i} \phi_{\mathrm{h}} / 4}=x_{\mathrm{h}}-$ $i(-1)^{i} y_{\mathrm{h}}$. Here, $\hat{x}$ and $\hat{y}$ are unit vectors in the $x$ and $y$ directions, $x_{\mathrm{s}}=\chi \cos \left(\phi_{\mathrm{s}} / 4\right), y_{\mathrm{s}}=\chi \sin \left(\phi_{\mathrm{s}} / 4\right)$, $x_{\mathrm{h}}=B \cos \left(\phi_{\mathrm{h}} / 4\right)$, and $y_{\mathrm{h}}=B \sin \left(\phi_{\mathrm{h}} / 4\right)$. The staggered flux state contains a density wave (particle-hole pairing) ordering whose symmetry is $d_{x^{2}-y^{2}}$, which is called " $d$ density wave." [28] The order parameters $y_{\mathrm{s}}$ and $y_{\mathrm{h}}$ correspond to the $d$-density-wave order parameter of spinons and holons, respectively. The mean-field Hamiltonian is diagonalized by the unitary transformation of the spinon and the holon. The obtained spectrum of fermions is $E_{\mathbf{k} \pm}= \pm \sqrt{\epsilon_{\mathbf{k}}^{2}+W_{\mathbf{k}}^{2}}-\mu$, where $\epsilon_{\mathbf{k}}=-X\left(\cos k_{x}+\cos k_{y}\right)$ and $W_{\mathbf{k}}=Y\left(\cos k_{x}-\cos k_{y}\right)$. We solved self-consistent equations numerically and the results are shown in the figures. The line of the $E_{\mathbf{k}-}=0$ is shown in Fig. 1. The upper band spectrum $E_{\mathbf{k}+}$ is always positive at finite doping where $\mu<0$.

In the present paper, we neglected $d$-wave RVB order because it was shown that the $d$-wave RVB order is completely destroyed by the $\mathrm{U}(1)$ gauge fluctuation above the BC temperature of the holons. [29] This means that the pseudogap region of the $t-J$ model, which is the underdoped region above the $\mathrm{BC}$ temperature, cannot be explained by the $d$-wave RVB state. We think that only possible state in this region is the staggered-flux state, which is equivalent to the $d$-wave RVB state at half-filling due to a SU(2) symmetry. [30] The reasons were discussed elsewhere. [22] It was shown by a variational Monte Carlo method that the ground state of the $t-J$ model near half filling is antiferromagnetic (AF) state. [31] However, in this paper we also neglected the possibilities of the AF state because it is known that experimentally there is no AF spin order in the pseudogap phase that we focused in this paper. We adopt the $t-J$ model where only the nearest-neighbor hopping term, often called the $t$ term, is included in the present paper. For a better description of actual high- $T_{c}$ superconductors, it is sometimes claimed that the next-nearest-neighbor hopping term, the $t^{\prime}$ term, is necessary. [32] We think that the $t^{\prime}$ term does not change the results qualitatively as long as the shape of the spectrum remains conical around $(\pi / 2, \pi / 2)$. In the staggered flux phase, it should be so. The growth of the Fermi arc near the transition point is sharp because our analysis is based on a mean-field theory. However, it is expected that the sharpness becomes weaker when the fluctuation around the mean-field solution is included.

In conclusion, we have analyzed doping and temperature dependences of the Fermi arc in the staggered flux ordered phase. Nontrivial behavior has been revealed. This behavior should be observed by precise experiments of ARPES in the pseudogap phase of high- $T_{\mathrm{c}}$ cuprates, if the pseudogap phase is the staggered-flux ordered phase.

K.H. thanks Masao Ogata, Youichi Yanase, Yoshifumi Morita, Takashi Koretsune, and Naokazu Shibata for their useful discussions. D.Y. appreciates hospitality of 
Aspen Center for Physics, where part of this work was done. Numerical computation in this work was partially carried out at the Yukawa Institute Computer Facility.

[1] X.-G. Wen and P. A. Lee, Phys. Rev. Lett. 76, 503 (1996); P. A. Lee, N. Nagaosa, T. K. Ng, and X.-G. Wen, Phys. Rev. B 57, 6003 (1998).

[2] S. Chakravarty, R. B. Laughlin, D. K. Morr, and C. Nayak, Phys. Rev. B 63, 094503 (2001).

[3] K. Machida and M. Ichioka, J. Phys. Soc. Jpn. 68, 2168 (1999); M. Ichioka and K. Machida, ibid. 68, 4020 (1999).

[4] A. V. Chubukov, D. K. Morr, and K. A. Shakhnovich, Philos. Mag. B 74, 563 (1996); A. V. Chubukov and J. Schmalian, Phys. Rev. B 57, R11085 (1998).

[5] V. J. Emery, S. A. Kivelson and O. Zachar, Phys. Rev. B 56, 6120 (1997).

[6] M. Randeria, Bose-Einstein condensation eds. A. Griffin, D. Snoke and S. Stringari (Cambridge, Cambridge University Press, 1994).

[7] B. Jankó, J. Maly and K. Levin, Phys. Rev. B 56, 11407 (1997).

[8] Y. Yanase and K. Yamada, J. Phys. Soc. Jpn. 68, 2999 (1999).

[9] D. Rohe and W. Metzner, Phys. Rev. B 63, 224509 (2001).

[10] S. Allen and A.-M. S. Tremblay, Phys. Rev. B 64, 075115 (2001).

[11] B. Kyung, S. Allen, and A.-M. S. Tremblay, Phys. Rev. B 64, 075116 (2001).

[12] I. Affleck and J. B. Marston, Phys. Rev. B 37, 3774 (1988); J. B. Marston and I. Affleck, ibid. 39, 11538 (1989).

[13] T. C. Hsu, J. B. Marston, and I. Affleck, Phys. Rev. B 43, 2866 (1991).
[14] H. A. Mook, P. Dai, and F. Dogan, Phys. Rev. B 64, 012502 (2001).

[15] H. A. Mook, P. Dai, S. M. Hayden, A. Hiess, J. W. Lynn, S.-H. Lee, and F. Dogan, Phys. Rev. B 66144513 (2002).

[16] R. I. Miller, R. F. Kiefl, J. H. Brewer, J. E. Sonier, J. Chakhalian, S. Dunsiger, G. D. Morris, A. N. Price, D. A. Bonn, W. H. Hardy, and R. Liang, Phys. Rev. Lett. 88, 137002 (2002).

[17] P. A. Lee and X.-G. Wen, Phys. Rev. B 63, 224517 (2001).

[18] H.-Y. Kee and Y. B. Kim, Phys. Rev. B 66, 012505 (2002).

[19] S. Chakravarty, Phys. Rev. B 66224505 (2002).

[20] M. U. Ubbens and P. A. Lee, Phys. Rev. B 46, 8434 (1992).

[21] K. Hamada and D. Yoshioka, Physica C 388 - 399, 80 (2003).

[22] K. Hamada and D. Yoshioka, Phys. Rev. B 67, 184503 (2003).

[23] For the most recent review article, see A. Damascelli, Z. Hussain, and Z-X. Shen, Rev. Mod. Phys. 75, 473 (2003).

[24] S. Chakravarty, C. Nayak, and S. Tewari, Phys. Rev. B 68, 100504 (2003)

[25] N. Nagaosa and P. A. Lee, Phys. Rev. Lett. 64, 2450 (1990); P. A. Lee and N. Nagaosa, Phys. Rev. B 46, 5621 (1992).

[26] G. Kotliar and J. Liu, Phys. Rev. B 38, 5142 (1988).

[27] Y. Suzumura, Y. Hasegawa, and H. Fukuyama, J. Phys. Soc. Jpn. 57, 2768 (1988).

[28] C. Nayak, Phys. Rev. B 62, 4880 (2000).

[29] M. U. Ubbens and P. A. Lee Phys. Rev. B 49, 6853 (1994).

[30] I. Affleck, Z. Zou, T. Hsu, and P. W. Anderson, Phys. Rev. B 38, 745 (1988).

[31] H. Yokoyama and M. Ogata, J. Phys. Soc. Jpn. 65, 3615 (1996).

[32] T. Tanamoto, H. Kohno and H. Fukuyama, J. Phys. Soc. Jpn. 63, 2739 (1994). 